\title{
A New High-Speed Shielded Curved Device Allowing Safe Posterior Thoracic Discectomy Through a Modified Transforaminal Thoracic Interbody Fusion Approach: Technique Description and Case Series
}

\author{
MICHAEL A. MILLGRAM, MD ${ }^{1}$ DANIEL E. KOLSKY, BSCMED,${ }^{1}$ WILLIAM J. BEUTLER, MD, ${ }^{2}$ \\ RICHARD D. GUYER, MD ${ }^{3}$ ELY ASHKENAZI, MD ${ }^{1}$ \\ ${ }^{I}$ Israel Spine Center, Assuta Hospital, Tel Aviv, Israel, ${ }^{2}$ Pennsylvania Spine Institute, Harrisburg, Pennsylvania, ${ }^{3}$ Texas Back Institute, Plano, Texas
}

\begin{abstract}
Background: The appropriate approach for surgical removal of thoracic disc herniations is controversial. The posterior approach historically acquired a bad reputation due to high rates of neurologic deterioration subsequent to spinal cord manipulation. The anterior approach has consequently gained popularity but entails a larger magnitude of surgery if open and is technically demanding if approached thoracoscopically. Approaching the thoracic disc posteriorly following unilateral facetectomy and pediculectomy was suggested in 1978. This study presents a technique for posterior unilateral thoracic discectomy through a hemilaminectomy, unilateral facetectomy, and hemipediculectomy, facilitated by a novel curved dorsally shielded high-speed device. Introducing the device ventral to the dural sac allows removal of calcified and soft disc fragments without relying on forceful manual maneuvers and avoiding manipulation of the spinal cord.
\end{abstract}

Methods: The maximal disc protrusion side is approached through a hemilaminectomy, unilateral facetectomy, and hemipediculectomy removing the superior half of the pedicle and exposing the disc transforaminally, allowing its removal using the device. Pedicle fixation and fusion concluded all procedures (TTIF). Between June 2014 and November 2018, 12 patients (6 men and 6 women) ages 23 to 74 years underwent posterior thoracic discectomy applying the above approach. The affected levels were D3 to D4 (1), D5 to D6 (1), D7 to D8 (1), D9 to D10 (1), D10 to D11 (3), D11 to D12 (4), and D12 to L1 (1).

Results: All patients presented with neurologic deterioration and all but 2 with pyramidal signs. All procedures were uneventful, without dural tears. None of the patients deteriorated neurologically. Average back pain visual analog scale scores decreased by 1.2 , from 6.6 to 5.4 . Average leg pain visual analog scale scores decreased by 2.2 , from 6.6 to 4.4. Improvement was noted in Oswestry Disability Index scores and 6 SF-36 metrics.

Conclusions: The new curved device and approach allow for a faster, safer thoracic disc herniation removal.

Clinical Relevance: The proposed technique allows a safer treatment for thoracic disc herniations, reducing complication rates and improving patient outcome.

New Technology

Keywords: discectomy, disc herniation, fusion, high-speed drill, posterior approach, thoracic

\section{INTRODUCTION}

Although the incidence of asymptomatic thoracic disc herniation (TDH) may be as high as $37 \%$, as shown in a magnetic resonance imaging (MRI) study on asymptomatic individuals, ${ }^{1}$ surgical treatment of TDH comprises between $0.15 \%$ and $4 \%$ of all disc operations. ${ }^{2,3}$ The improvement in diagnostic imaging abilities has led to more frequent and early-stage diagnosis of thoracic disc herniation. Removal of the disc is required when neurologic deterioration occurs secondary to spinal cord compression.

The approach to thoracic disc excision remains controversial. The posterior approach, although familiar to most spine surgeons, lost favor when in a high percentage of surgeries neurologic deterioration occurred. ${ }^{4}$ The transpedicular approach and the costotransversectomy approach allow posterolateral access by removing the facet joint and pedicle ipsilateral to the lesion ${ }^{5,6}$ or by removing a short segment of rib and the adjacent transverse process, ${ }^{7}$ respectively. These approaches provide posterolateral 
access to the spinal canal, but the removal of disc material, often calcified, requires forceful manual maneuvers that can jeopardize the spinal cord.

Lateral extracavitary approaches were reported by several groups, ${ }^{8-10}$ involving resection of a longer segment of the rib and pleural retraction, in order to provide better access and visibility. Another similar option is the transthoracic approach, in which the thoracic disc is exposed by anterolateral thoracotomy. ${ }^{8-11}$ However, this approach is of large magnitude and has a higher pulmonary complication rate. ${ }^{10}$ Thorascopic techniques ${ }^{12,13}$ and other minimally invasive approaches reduce tissue damage, leading to reduced pain and shorter hospitalization. ${ }^{6,10,14,15}$ However, these techniques are technically demanding, expensive, and require extensive training, ${ }^{14,16,17}$ and may result in less favorable clinical results.

The unilateral facetectomy and pediculectomy approach was first described in 1978..$^{5}$ The use of this relatively easier approach would be desirable while increasing the safety and reducing the length of the procedure. Transforaminal thoracic interbody fusion (TTIF) was first reported in 2010 as a potential approach to treat TDH. ${ }^{18}$ This method is useful for patients with a lateral type of disc herniation. However, its application to central disc herniation is limited. In addition, the approach is limited by the narrower distance between the ipsilateral pedicles, especially at the upper thoracic spine levels. Therefore, it is more difficult to achieve safe decompression at the upper thoracic spine with TTIF. ${ }^{19}$

This study aims to present the authors' experience with a new high-speed shielded curved device facilitating posterior thoracic discectomy through a modified TTIF approach, including a hemilaminectomy unilateral facetectomy and hemipediculectomy, allowing the convenience of the traditional posterior approach with a neurologically safer and shorter procedure.

\section{MATERIALS AND METHODS}

Institutional Review Board approval was obtained for medical record review. The CARE guidelines for clinical case reports and case series were used for the preparation of this study. ${ }^{20}$

\section{Surgical Tool Design}

The DReal curved dorsally shielded high-speed device (Carevature Medical Ltd, Rehovot, Israel) is

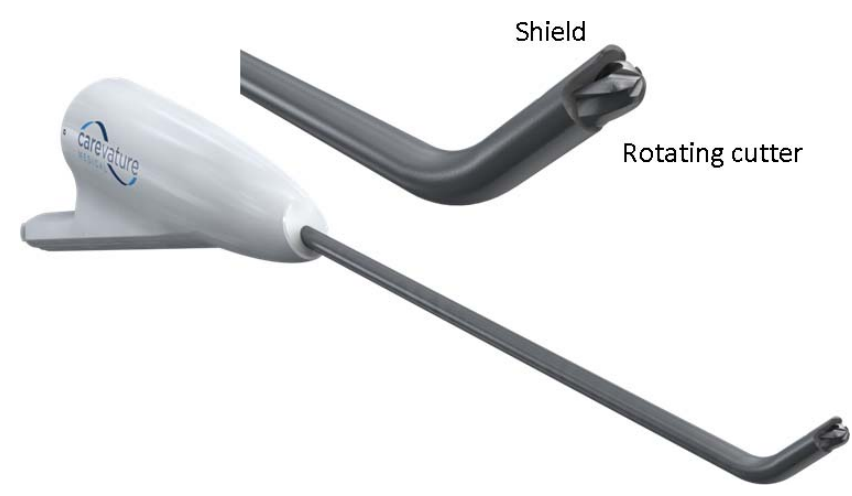

Figure 1. The DReal (lower left) and expanded view of the distal tip (upper right).

a disposable product with a cutting head $3 \mathrm{~mm}$ in diameter, angled at 45 degrees, rotating at up to $60000 \mathrm{rpm}$, and shielded dorsally (Figure 1). It is powered by an electric 80-Watt surgical motor of various vendors and has a built-in irrigation channel connecting to a standard $0.9 \% \mathrm{NaCl}$ infusion bag and intravenous fluid tubing, allowing continuous irrigation and cooling of the device head.

\section{Surgical Approach and Technique}

The pathologic area is identified using fluoroscopic imaging and exposed posteriorly including the 2 adjacent levels. A hemilaminectomy and total facetectomy is undertaken on the side of maximal disc protrusion or, if central, the side of most profound motor deficit. Using a standard highspeed drill or box chisel, a hemipediculectomy is performed, removing the superior half of the pedicle adjacent to the disc space. A transforaminal annulotomy and disc space evacuation is performed using pituitary rongeurs. As Figure 2 shows, the curved dorsally shielded high-speed device is then inserted into the disc space and completes removal of disc material from the space, exposing the bony surface of the end plates. The anterior edge of the device is then used to drill away posterior subannular disc and calcified tissue, dorsally progressing until the posterior vertebral body border. Figure 3 shows an intraoperative fluoroscopic image of the device inside the disc space. Figure 4 shows the device from the surgeon's perspective, inside and outside the disc space.

Using delicate curved, down-facing curettes and an angled Ray dissector the dura is gently separated from the protruded disc material, which is then depressed into the vacated disc space and finally removed with pituitary rongeurs. The disc space is 


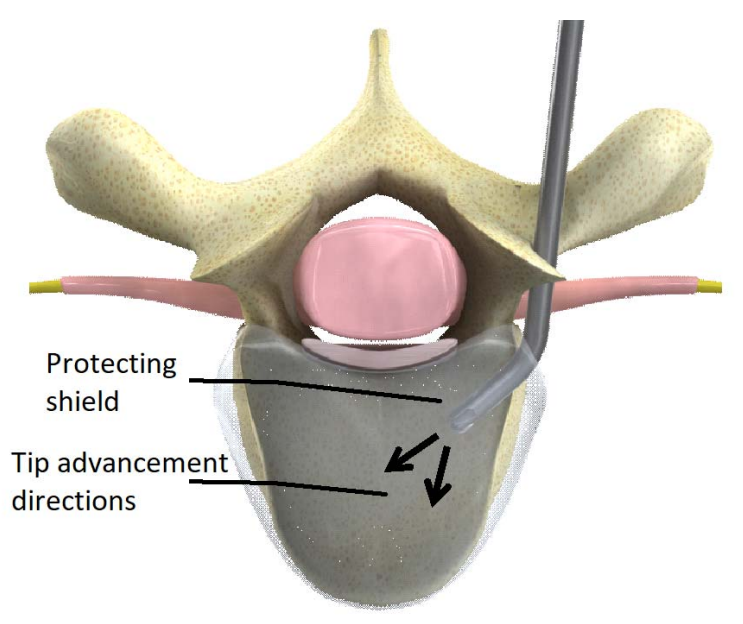

a

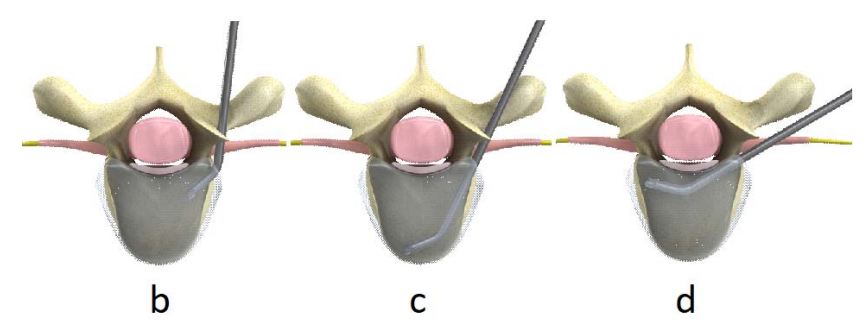

Figure 2. (a) Safety feature of the protective shield. (b-d) Navigating inside the disc space.

then packed with local autologous bone, and the disc space may be supported with an intradiscal cage. The procedure is concluded with a posterior pedicle screw fixation and contralateral posterior fusion.

\section{Study Population}

Twelve above procedures were performed in our service between June 2014 and November 2018. Patients included 6 men and 6 women, ages 23 to 74 years. The levels operated on were D3 to D4 (1), D5

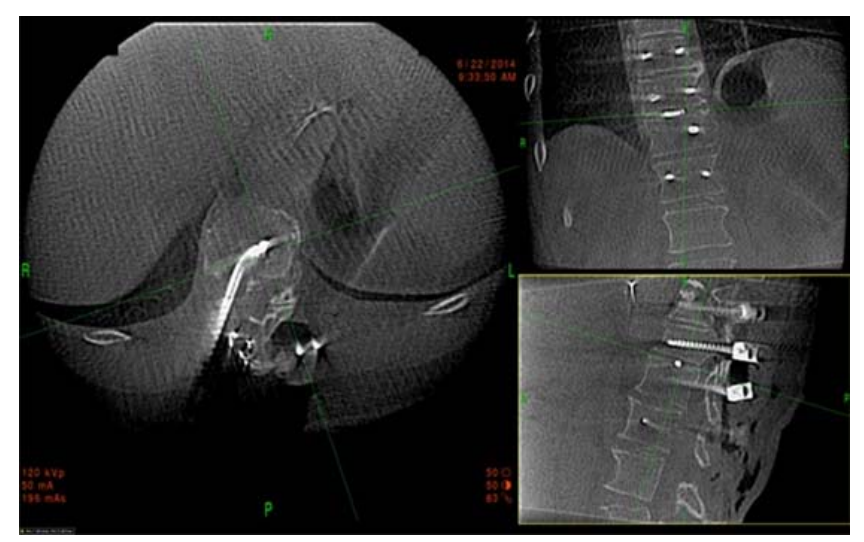

Figure 3. Intraoperative fluoroscopic image of the DReal inside the disc space.
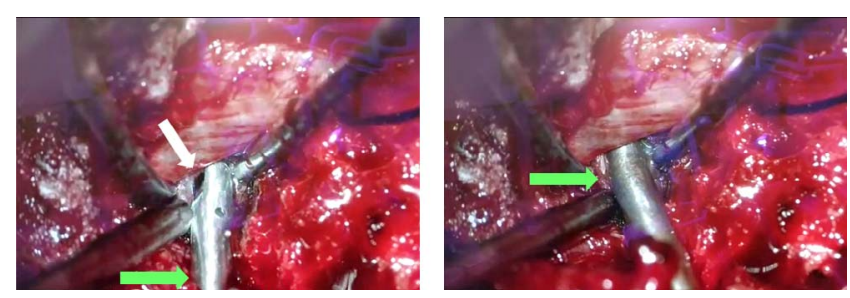

Figure 4. Left: The device outside the disc space; the drill side is marked with a white arrow. Right: The device in the disc space underneath the cord. The curved region is marked with a green arrow in both pictures.

to D6 (1), D7 to D8 (1), D9 to D10 (1), D10 to D11 (3), D11 to D12 (4), and D12 to L1 (1). All patients presented with neurologic deterioration and all but 2 with pyramidal signs (1 D11 to D12 and 1 D12 to L1).

In all surgeries polymodal somatosensory and transcranial motor potentials were monitored. Intraoperative O-arm (Medtronic, Minneapolis, Minnesota) axial sagittal and coronal computed tomography (CT) images verified screw and cage placement and disc removal.

The clinical outcomes were assessed preoperatively and at the final follow-up using visual analog scale, Oswestry Disability Index, and SF-36 questionnaires. Data were analyzed only for patients who had both preoperative and postoperative questionnaires $(\mathrm{n}=5)$.

\section{RESULTS}

\section{Case Reports}

\section{Case 1}

A 62-year-old otherwise healthy woman presented with a 4-year history of right lower limb pain and recent onset of left lower limb pain and bilateral leg weakness. The physical examination was positive for bilateral leg weakness in all distributions of 3-4/ 5, an atactic gait, and bilateral Babinski signs. MRI revealed a disc herniation with cord compression at the D11 to D12 level. The surgery as described above was approached from the right side, and a D10 to L1 instrumented fusion was undertaken to address local kyphosis. Leg pain resolved, and leg weakness and ambulation improved at more than 3year follow-up.

\section{Case 2}

A 39-year-old otherwise healthy man presented with progressive left leg weakness and back pain. Physical examination revealed proximal left leg weakness 3/5. MRI revealed a disc herniation at 
the D11 to D12 level, more to the right with cord compression, local kyphosis, and a high-intensity signal in the spinal cord at the D11 to D12 level. The surgical procedure described was performed from the right, and instrumented fusion D11 to L1 to address the kyphosis completed the procedure. Leg weakness is improved at more than 3-year followup.

\section{Case 3}

A 51-year-old woman with an unremarkable medical history other than smoking and an ACDF C4 to C5 in 2012 and a C6 corpectomy and ASF C5 to C7 in 2013 presented with right leg pain and paraesthesias. Physical examination was remarkable for a bilaterally positive Babinski response, hyperactive reflexes in the right leg, and mild right leg weakness $4+/ 5$. MRI revealed a calcified disc herniation mostly on the right and cord compression at the D12 to L1 level. A central L1 to L2 disc protrusion was also evident. The surgical procedure described was undertaken at the D12 to L1 level from the right. An instrumented fusion was then performed D12 to L2 to address the degenerative level L1 to L2. At 2.5-year follow-up she is pain free, with full motor power in her legs.

\section{Case 4}

A 44-year-old man with a past history of ankylosing spondylitis, posterior L1 to L2 fusion due to fracture, diabetes mellitus type 2, obesity, and hypertension presented with back pain and progressive weakness in both legs during the 2 years prior to the surgery. His physical examination revealed a short neck, significantly reduced neck motion, a barrel chest, hyperactive reflexes in the legs, a positive Babinski sign on the left, and a spastic gait. MRI revealed ankylosing spondylodiscitis at the D11 to D12 level, with cord compression of disc and bony tissue in the canal more on the right. The described procedure was undertaken at D11 to D12 from the right. A 13-mm intradiscal PEEK cage was inserted, and a long posterior fusion D8 to L3 using expanding head screws was applied because of ankylosis and poor bone quality. At 2.5-year follow-up his pain is resolved. Neurologic status remains unchanged.

\section{Case 5}

A 34-year-old man with an unremarkable medical history other than smoking presented with a 3-year history of thoracic back pain and recent-onset left leg pain and weakness. Physical examination revealed hyperactive reflexes in the legs and significant $3 / 5$ left leg weakness. MRI revealed a calcified D5 to D6 disc herniation to the right. The described procedure was undertaken from the right. A 3-mm PEEK cervical cage was inserted in the disc space, and a contralateral D5-6 instrumented fusion was performed. At 2-year follow-up there is improvement in both pain and left leg motor power.

\section{Case 6}

A 23-year-old healthy woman with a past history of smoking presented with upper thoracic back pain and right leg weakness 2 years following a motor vehicle accident. The pain and weakness began with the accident and had persisted unchanged. The right leg had difficulty supporting her weight, and she suffered recurrent falls. The examination revealed a Babinski response on the left, a high thoracic sensory level, and right leg weakness. On thoracic MRI, a D3 to D4 central disc herniation compressing the spinal cord and a D4 hemangioma were appreciated. The surgery as described above was approached from the right. PMMA bone cement was injected into the D4 hemangioma to support a bone screw, and bone screws were inserted on the left D3 to D4, and a left posterior fusion was completed. A cervical PEEK cage was inserted in the evacuated D3 to D4 disc space from the right. Leg weakness improved after surgery.

\section{Case 7}

A 44-year-old otherwise healthy woman presented with thoracic back pain, paraesthesias, and numbness in both legs. A right herniated disc was observed on MRI at the D7 to D8 level, and a disc bulge at D9 to D10 was also demonstrated with associated spinal stenosis. A bilateral positive Babinski sign was elicited together with hyperactive reflexes in the left leg.

The above-described right thoracic discectomy was performed at the D7 to D8 level, and a laminectomy at the D9 to D10 level. A posterior instrumented fusion D7 to D10 completed the procedure. Leg paraesthesias resolved and numbness improved postoperatively without change at 2.5-year follow-up.

\section{Case 8}

A 51-year-old woman with a past history of psoriasis and lumbar decompression and fusion presented with thoracic back pain and bilateral leg 
weakness. MRI revealed a herniated disc at the D10 to D11 level with cord compression. Examination confirmed bilateral leg weakness and revealed hyperactive reflexes in both legs.

The above-described procedure was performed from the left. An intervertebral cage was then inserted and a posterior instrumented fusion undertaken at D10 to D11. Leg weakness as well as back pain improved postoperatively and remained stable at 1 year.

Figure 5 shows the preoperative and postoperative MRI scans of the patient. Cord compression is evident prior to the procedure and it was relieved at the postoperative scan, taken approximately a year after the surgery.

\section{Case 9}

A 74-year-old man with a past history of hypertension, cervical and lumbar fusion operations, and sleep apnea was referred because of bilateral spastic leg weakness, which progressed, and at presentation he was unable to stand without support. Examination revealed bilateral profound leg weakness, bilateral Babinski responses, and hyperactive reflexes in the legs. MRI scans demonstrated a herniated disc D9 to D10 central and right with cord compression and myelomalacia of the cord. A right-sided approach and discectomy as described above were undertaken, and an intervertebral cage was inserted. A posterior fusion D9 to D10 completed the procedure. After decompression, motor-evoked potentials, which were absent at the initiation of the procedure, appeared in both quadriceps and left tibialis anterior. Postoperative leg weakness improved, but the patient still requires a walker to ambulate 1 year postoperatively.

\section{Case 10}

A 38-year-old otherwise healthy woman fell from height 3 years prior to presentation, with a fracture of D12, posttraumatic kyphosis, and a bony fragment on the spinal canal compressing the spinal cord at the D11 to D12 level. Associated fractures were of the right calcaneus, right tibia, and left hip. She developed a neurogenic bladder with left hydronephrosis, and she performs self-catheterization. Right leg weakness also followed the injury. On examination a right leg severe dorsiflexor and plantar flexor weakness was documented, and she ambulates with an ankle-foot orthosis but mostly is wheelchair bound. Left quadriceps weakness also
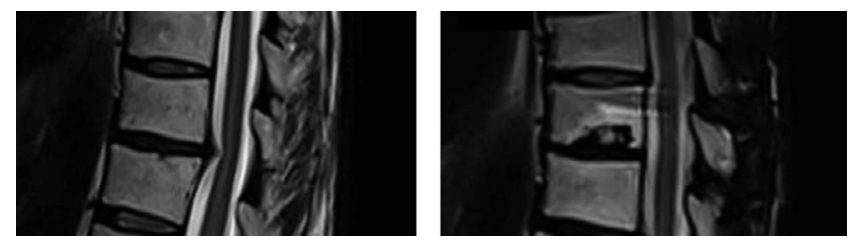

Figure 5. Preoperative (left) and postoperative (right) magnetic resonance imaging scans of patient no. 8, experiencing disc herniation at D10 to D11.

was found. A left thoracic D11 to D12 resection of the bony mass applying the presented drill and approach and posterior fusion D10 to D12 were performed. Both ambulation and motor power in both legs were improved at 3-month follow-up.

\section{Case 11}

A 71-year-old man with a past history of bronchial asthma, myocardial infraction, and subsequent catheterization presented with progressive bilateral paraparesis and required a wheelchair to ambulate. CT and MRI revealed severe D10 to D11 stenosis and disc herniation, cord compression, and a pathologic signal in the spinal cord, as well as lumbar stenosis and kyphosis. Physical examination revealed bilateral spastic leg weakness, full paralysis of the left foot, quadriceps weakness $(2 / 5$ on the left and $4 / 5$ on the right), bilateral Babinski responses, and hyperactive reflexes in the legs. The patient was unable to walk and experienced occasional urinary incontinence. The described procedure was performed at D10 to D11 with a bilateral D11 laminectomy, partial right pediculectomy, and removal of ossified ligament and cyst adherent to the dural sac. A posterior instrumented fusion at D10 to D12 completed the procedure. At the initiation of the procedure, motor-evoked potentials were absent below the iliopsoas, including in the sphincter muscles. After the decompression, motorevoked potentials appeared from the left sphincter muscle and left foot. At 3-month follow-up, the patient was able to move the left foot and walk with a walker. The urinary incompetence was resolved.

\section{Case 12}

A 45-year-old man was referred because of progressive leg weakness and paraesthesias. The patient was a heavy smoker with a history of left orchiectomy due to seminoma, bariatric surgery, chronic obstructive pulmonary disease, hyperlipidemia, lithotripsy, and cluster headaches. CT and MRI revealed a left D10 to D11 herniated disc with cord compression and myelomalacia. Substantial left 
Table. Patient outcome metrics.

\begin{tabular}{|c|c|c|c|}
\hline Scale & Preoperative & Postoperative & Difference \\
\hline \multicolumn{4}{|l|}{ VAS } \\
\hline Back pain & 6.6 & 5.4 & -1.2 \\
\hline Leg pain & 6.6 & 4.4 & -2.2 \\
\hline ODI & 42.67 & 35.51 & -7.16 \\
\hline \multicolumn{4}{|l|}{ SF-36 } \\
\hline Physical functioning & 45 & 49 & +4 \\
\hline $\begin{array}{l}\text { Role limitations due to } \\
\text { physical health }^{\mathrm{a}}\end{array}$ & 8.33 & 41.67 & +33.33 \\
\hline $\begin{array}{l}\text { Role limitations due to } \\
\text { emotional problems }\end{array}$ & 0 & 44.44 & +44.44 \\
\hline Energy/fatigue $^{\mathrm{a}}$ & 41.67 & 33.33 & -8.33 \\
\hline Emotional well-being ${ }^{\mathrm{a}}$ & 48 & 68 & +20 \\
\hline Social functioning $^{\mathrm{a}}$ & 58.33 & 79.17 & +20.83 \\
\hline Pain & 37 & 50 & +13 \\
\hline General health & 66.25 & 55.75 & -10.5 \\
\hline
\end{tabular}

Abbreviations: ODI, Oswestry Disability Index; VAS, visual analog scale.

${ }^{\text {a}}$ Postoperative data were available for 3 patients.

quadriceps weakness was found at the physical examination. The surgical procedure described was performed from the left at D10 to D11 and was concluded with a D10 to D11 fusion. After 3 months, leg weakness and sensation were improved.

\section{Clinical Outcome}

The average VAS, Oswestry Disability Index, and SF-36 scores appear in the Table. As the Table demonstrates, improvement was obtained in most parameters (all but the SF-36 energy/fatigue and general health scores), indicating that patient pain and disability were reduced.

\section{DISCUSSION}

Performing an anterior thoracic discectomy either thorascopically or open is technically demanding and may be associated with increased morbidity. Allowing a safe, familiar, less morbid posterior approach is desirable.

The DReal curved, dorsally shielded high speed device can facilitate a limited posterior approach and provide a safe method of disc removal and decompression. The curved configuration of the device allows access to the disc space without rib head removal and extending the approach laterally and remaining transforaminal. Hemipediculectomy allows better visualization and working space. Continuous irrigation of the device head precludes thermal damage to the tissues.

This study is limited by the small series of patients from a single institution. Outcome metrics were only partially available because most of the patients did not complete either the preoperative questionnaire or the postoperative questionnaire. Future studies will include questionnaire completion at follow-up examinations to reduce the risk of patient unavailability for follow-up questionnaires. However, our experience shows that this technique can significantly improve the clinical outcome.

\section{CONCLUSION}

The DReal curved dorsally shielded high-speed irrigated device can facilitate a safe limited posterior exposure thoracic discectomy. This concept is supported by this small series.

\section{REFERENCES}

1. Wood KB, Garvey TA, Gundry C, Heithoff KB. Magnetic resonance imaging of the thoracic spine: evaluation of asymptomatic individuals. $J$ Bone Joint Surg Am. 1995;77(11):1631-1638.

2. Arce CA, Dohrmann GJ. Herniated thoracic disks. Neurol Clin. 1985;3(2):383-392.

3. Arseni C, Nash F. Thoracic intervertebral disc protrusion: a clinical study. J Neurosurg. 1960;17(3):418-430.

4. McCormick WE, Will SF, Benzel EC. Surgery for thoracic disc disease: complication avoidance: overview and management. Neurosurg Focus. 2000;9(4):1-6.

5. Patterson Jr RH, Arbit E. A surgical approach through the pedicle to protruded thoracic discs. J Neurosurg. 1978;48(5):768-772.

6. Lesoin F, Rousseaux M, Autricque A, et al. Thoracic disc herniations: evolution in the approach and indications. Acta Neurochir (Wien). 1986;80(1):30-34.

7. Hulme A. The surgical approach to thoracic intervertebral disc protrusions. J Neurol Neurosurg Psychiatry. 1960;23(2):133.

8. Maiman DJ, Larson SJ, Luck E, El-Ghatit A. Lateral extracavitary approach to the spine for thoracic disc herniation: report of 23 cases. Neurosurgery. 1984;14(2):178-182.

9. Foreman PM, Naftel RP, Moore TA, Hadley MN. The lateral extracavitary approach to the thoracolumbar spine: a case series and systematic review. J Neurosurg Spine. 2015;24(4):570-579.

10. Mulier S, Debois V. Thoracic disc herniations: transthoracic, lateral, or posterolateral approach?: a review. Surg Neurol. 1998;49(6):599-608.

11. Sekhar LN, Jannetta PJ. Thoracic disc herniation: operative approaches and results. Neurosurgery. 1983;12(3):303-305.

12. Rosenthal D, Rosenthal R, de Simone A. Removal of a protruded thoracic disc using microsurgical endoscopy: a new technique. Spine. 1994;19(9):1087-1091.

13. Regan JJ, Mack MJ, Picetti III GD. A technical report on video-assisted thoracoscopy in thoracic spinal surgery: preliminary description. Spine. 1995;20(7):831-837.

14. Khoo LT, Beisse R, Potulski M. Thoracoscopic-assisted treatment of thoracic and lumbar fractures: a series of 371 consecutive cases. Neurosurgery. 2002;51(suppl 2):S2-S104.

15. Perez-Cruet MJ, Kim BS, Sandhu F, Samartzis D, 
Fessler RG. Thoracic microendoscopic discectomy. J Neurosurg Spine. 2004;1(1):58-63.

16. Yoshihara H. Surgical treatment for thoracic disc herniation: an update. Spine. 2014;39(6):E406.

17. McAfee PC, Regan JR, Zdeblick T, et al. The incidence of complications in endoscopic anterior thoracolumbar spinal reconstructive surgery: a prospective multicenter study comprising the first 100 consecutive cases. Spine. 1995;20(14):16241632.

18. Machino M, Yukawa Y, Ito K, Nakashima H, Kato F. A new thoracic reconstruction technique "transforaminal thoracic interbody fusion": a preliminary report of clinical outcomes. Spine. 2010;35(19):E1000-E1005.

19. Yamasaki R, Okuda S, Maeno T, Haku T, Iwasaki M, Oda T. Surgical outcomes of posterior thoracic interbody fusion for thoracic disc herniations. Eur Spine $J$. 2013;22(11):2496-2503.

20. Gagnier JJ, Kienle G, Altman DG, et al. The CARE guidelines: consensus-based clinical case report guideline development. J Clin Epidemiol. 2014;67(1):46-51.

Disclosures and COI: M.A.M. and R.G. are consultants for Carevature Medical Ltd, which manufactures the device mentioned in the study. E.A. is a shareholder of and consultant for Carevature Medical Ltd, which manufactures the device mentioned in the study. This research received no specific grant from any funding agency in the public, commercial, or not-for-profit sectors.

M.A.M and D.K. contributed equally to this study.

Corresponding Author: Michael A. Millgram, MD, Israel Spine Center, Assuta Hospital, Habarzel 20, Tel Aviv 69710, Israel. Phone: (972)546319458; Email: research@isc.co.il.

Published 31 December 2019

This manuscript is generously published free of charge by ISASS, the International Society for the Advancement of Spine Surgery. Copyright $(\subset 2019$ ISASS. To see more or order reprints or permissions, see http://ijssurgery.com. 